family doctors and in a few instances by psychiatrists. The most common cause of death was poisoning, which accounted for 57 of those diagnosed as mentally ill. In about half the cases, whether classified as mentally ill or not, the blood alcohol concentration was raised.

Overall the findings seem strongly to favour that a substantial number of cases of accidental death are concealed suicides. The investigators were, however, rightly cautious in not pushing their conclusions too far. What they did conclude, and this seems to be indisputable, is that, like undetermined deaths, accidental deaths-especially those due to selfpoisoning, solitary drowning, and falls from a height-should be included in the mortality of mental illness.

1 British Medical fournal, 1977, 1, 128

2 World Health Organisation, Suicide and Attempted Suicide, Public Health Papers No 58, ed Eileen M Brooke. Geneva, World Health Organisation, 1974.

${ }^{3}$ Holding, T A, and Barraclough, B M, British fournal of Psychiatry, 1977, $130,244$.

${ }^{4}$ Holding, T A, and Barraclough, B M, British fournal of Psychiatry, 1975, $127,133$.

\section{Late infection after total hip replacement}

Infection is the most serious complication of the replacement of a joint-and the only one likely to lead to the permanent removal of the prosthesis itself and hence to total loss of function. Published reports dealing with the incidence of infection have been confused by a failure to distinguish among four different sets of circumstances. These are, firstly, loosening of the prosthesis, combined with the formation in and around the implant of pultaceous material with the appearance of pus but which on culture proves to be sterile; secondly, strictly superficial sepsis soon after operation, which may be treated without prejudicing the implant itself; thirdly, deep infection around the implant itself soon after operation; and, fourthly, the development of bacteriologically proved infection around the implant itself one or more years after operation (late infection).

In some studies loosening of the prosthesis associated with the presence of sterile, pus-like material has been taken as being due to infection. The sterility has been attributed to the effect of antibiotics used postoperatively. Alternatively the explanation offered has been the presence of anaerobic organisms or L-forms not usually regarded as pathogens and therefore neither sought nor detected bacteriologically. These views have led to the introduction into orthopaedic terminology of the term "sterile infection." Though possibly the loosening and the presence of material resembling pus in the absence of cultured bacteria are accounted for by one of these postulated mechanisms, the presence of infection is not certain, since Koch's postulates are not satisfied. Furthermore, we now know that the tissue response to the abrasion debris produced from polymethylmethacrylate when a prosthesis loosens will lead to necrosis-and hence to the presence of pus-like material around the prosthesis-while local sensitivity to the implant may also lead to necrosis, loosening of the prosthesis, and the formation of pultaceous matter. ${ }^{1}$

Clearly true superficial infections should be included in data on the rate of postoperative sepsis. Nevertheless, generally speaking their occurrence has no bearing upon the long-term outcome of the operation. Infection has an important role when it is bacteriologically proved and occurs around the prosthesis itself either early or late postoperatively. To reduce the incidence of these infections Charnley pioneered the use of a special operating environment aimed at reducing the bacteriological contamination of the air around the wound, and perhaps because of this early sepsis rates have now fallen in all good orthopaedic centres to negligible levels. In fact zero or near-zero early sepsis rates can be achieved in joint replacement surgery without the use of special techniques for sterilising the ambient air, ${ }^{2}$ and a recent bacteriological study of theatre air and the wound during joint replacement has suggested that, provided the contamination of the air is reduced below a certain threshold, no organisms can be isolated from the wound during operation. ${ }^{3}$

In Charnley's experience the incidence of late infection fell in parallel with that of early infection, and he concluded, therefore, that late infection was due to the introduction of organisms around the prosthesis at the time of surgery, which then remained dormant for several years. ${ }^{45}$ Clearly this is possible: we know-for example, in osteomyelitis-that organisms may remain dormant in this way in bone. On the other hand, it is difficult to believe that spread to the implant through the blood stream never occurs, especially in rheumatoid arthritis, in which spontaneous septic arthritis is well recognised. The likelihood that the infection reached the joint by the haematogenous route becomes greater as the interval between implantation and the development of sepsis increases. Thus most of Charnley's delayed infections occurred within 18 months and may well, therefore, have been due to the mechanism which he describes. Conversely infection has occurred up to five years after inplantation, ${ }^{3}$ and in several recent studies extremely strong (though ultimately circumstantial) evidence has been produced in support of the haematogenous route ${ }^{6-10}$ These studies have shown, for example, that the same organism was cultured from the blood and from the joint that was infected later, or that two joints became infected with the same organism. Putting these data together, the conclusion seems inescapable that haematogenous infection can occur in a replaced joint-which is not to say that every case of late infection is haematogenous.

If the possibility of haematogenous infection is accepted, two important conclusions may be drawn. Firstly, modifications of the operating environment cannot be expected to reduce the late sepsis rate to nil, and any assessment of the efficacy of these techniques based on late sepsis rates may be misleading. Secondly, and of greater importance for doctors in general, any patient who has had a joint replaced must be treated by an appropriate prophylactic antibiotic umbrella should he develop any other intercurrent illness likely to result in bacteraemia. Of themselves, such illnesses may be trivial, but their consequences may be disastrous if the replaced joint is lost.

${ }^{1}$ Evans, E M, et al, fournal of Bone and foint Surgery, 1974, 56B, 626.

2 Collis, D K, 43rd Annual Meeting, American Academy of Orthopaedic Surgeons, New Orleans, 1976.

3 Freeman, M A R, et al, Archiv für Orthopädische und Unfall-Chirurgie, 1977 , in press.

4 Charnley, J, Clinical Orthopaedics and Related Research, 1972, 87, 167.

5 Charnley, J, and Eftekhar, N, British fournal of Surgery, 1969, 56, 641.

${ }^{6}$ Burton, D S, and Schurman, D J, fournal of Bone and foint Surgery, 1975, 57A, 1004.

${ }^{7}$ Creuss, R L, Bickel, W S, and von Kessler, K L C, Clinical Orthopaedics and Related Research, 1975, 106, 99.

${ }^{8}$ Mallory, T H, Fournal of Bone and foint Surgery, 1973, 55A, 1753.

${ }^{9}$ Hall, A J, Fournal of Bone and foint Surgery, 1974, 56B, 144.

${ }_{10}$ Downes, E M, Fournal of Bone and foint Surgery, 1977, 59B, 42. 